\title{
Efficacy of Different Image Processing Programs in Detecting Small Artificial Caries Defects on Approximal Surfaces
}

\author{
Weeraya Tantanapornkul ${ }^{1 *}$, Preeyanit Mongkolrop ${ }^{2}$, Paweena Manoping ${ }^{2}$, Achara Hannanta-anant ${ }^{2}$ and Ekkarin Prompruk ${ }^{2}$
}

${ }^{1}$ Instructor, Department of Oral Diagnosis, Faculty of Dentistry, Naresuan University, Thailand

${ }^{2}$ Sixth grade dental student, Faculty of Dentistry, Naresuan University, Thailand

\begin{abstract}
Objectives: To compare the efficacy of different image processing algorithms of three digital x-ray software programs in detecting small artificial approximal caries defects.

Methods: 75 extracted human maxillary and mandibular posterior permanent teeth were selected. The 0.5 millimeter diameter high speed diamond bur was used to prepare and simulate small dental caries on proximal surfaces of the teeth. The teeth were mounted in 25 plaster blocks. Each of them contained 2 premolars and 1 molar. A prominent part of proximal surfaces were placed at the same vertical level to simulate normal anatomical contacts. Bitewing radiographs were taken and viewed with Vixwin 200, Dimaxis and Dr. Suni plus. The radiograph was enhanced with emboss, gray-scale reversed and contrast-brightness tools. Three observers assessed the digital radiographs from each modality for a presence or an absence of small proximal carries. The sensitivity and specificity of 3 digital x-ray software programs were compared by McNemar test. The intraobserver and interobserver agreement were analyzed with Kappa statistic analysis.
\end{abstract}

Results: The sensitivity and specificity of emboss enhancement of Vixwin 2000, Dimaxis and Dr. Suni plus were $50.00 \%, 68.00 \%, 42.00 \%$ and $92.00 \%, 86.00 \%, 96.00 \%$ respectively. The sensitivity and specificity of grayscale reverse enhancement from the same company were $89.00 \%, 89.00 \%, 89.00 \%$ and $92.00 \%, 92.00 \% 90.00 \%$ respectively. The sensitivity and specificity of contrast-brightness enhancement from the same company were $91.00 \%$, $81.00 \%, 84.00 \%$ and $86.00 \%, 92.00 \%, 92.00 \%$, respectively.

Conclusions: The efficiency of Dimaxis's emboss enhancement digital radiograph was higher than Vixwin 2000 and Dr. Suni plus $(P<0.05)$. No significant difference of gray-scale reverse and contrast-brightness's enhancement efficiency $(P>0.05)$.

\section{Introduction}

The rapid advances in computer technology have had a significant impact on dental radiography. With digital intraoral radiography introduced to dentistry, many studies have been performed to validate the reliability and reproducibility of digital intraoral radiographs for caries detection, especially proximal caries [1-5]. Several digital radiographic systems are currently used in dentistry instead of filmbased radiography, such as Emago (Emago Dental software, ODS, Amsterdam, The Netherlands), Digora Optime (Soredex, Helsinki, Finland), Vixwin 2000 (Gendex $^{\circledR}$ Visualix $^{\circledR}$ eHD; Gendex Dental Systems, Des Plaines, IL, USA), Dimaxis (Planmeca Dixi 3, Planmeca Oy, Helsinki, Finland), and Dr. Suni Plus (Suni Medical Imaging Inc. , California, USA). Many studies have shown that direct digital systems have many advantages over conventional film $[2,6,7]$, such as reduced exposure dose and exposure time (no need for film processing). There are two different approaches to direct digital image acquisition: the solid state-based system (charge-coupled device (CCD) and complementary metal oxide semiconductor (CMOS) active pixel image sensors) and the photo-stimulable phosphor (PSP) plate systems. It has been accepted that the diagnostic accuracy of the CCD-based system is also equivalent to that of conventional intraoral radiography for detecting caries [1]. Image enhancements such as contrast and brightness adjustment, color-coded, subtraction, gray-scale reversed, or emboss modulation are the other advantages of digital imaging, which may increase diagnostic accuracy $[2,6-8]$.

The greatest difficulty in caries detection concerns the carious lesion in its initial stages especially in the thick crown and large size of proximal surfaces such as posterior teeth $[3,9]$. Therefore small carious lesion especially in proximal surfaces has traditionally been diagnosed by clinical examination combined with radiography. Available direct digital systems differ in their software capability
[10]. Image processing enhancement programs offer various image appearances, which mostly require subjective measures for diagnosis. Various digital systems display the images in the systems' own software, which often differ significantly in relation to design, userfriendliness, number and quality of facilities for image enhancement. During the past years, the accuracy of different digital radiography systems for caries detection has been compared mutually and with conventional film systems [11-14]. However, these studies focused on the efficacy between the programs within the same digital systems. To our knowledge, there have been few studies concerned the efficacy of digital image enhancement programs between the different systems in detecting small carious lesions. Gotfredsen et al. [15] evaluated observers' use of image-enhancement facilities and time consumption in assessing approximal and occlusal caries in radiographs taken with four digital systems. They concluded that the observers took advantage of the facilities available for enhancement of density and contrast in digital images. Hintze [16] compared the caries diagnostic accuracy of two software modalities used in the assessment of digital radiographs obtained with four different dental systems. He concluded that there

*Corresponding author: Weeraya Tantanapornkul, Department of Oral Diagnosis, Faculty of Dentistry, Naresuan University, Phitsanulok 65000 Thailand, Tel: 66-55966-955; Fax: 66-55-966-035; E-mail: weeraya_t@yahoo.com

Received March 24, 2012; Accepted March 26, 2012; Published March 28, 2012 Citation: Tantanapornkul W, Mongkolrop P, Manoping P, Hannanta-anant A, Prompruk E (2012) Efficacy of Different Image Processing Programs in Detecting Small Artificial Caries Defects on Approximal Surfaces. Dentistry 2:129. doi:10.4172/2161-1122.1000129

Copyright: (c) 2012 Tantanapornkul W, et al. This is an open-access article distributed under the terms of the Creative Commons Attribution License, which permits unrestricted use, distribution, and reproduction in any medium, provided the original author and source are credited. 
was no significant difference in caries diagnostic accuracy between two software modalities used for examination of digital radiographs obtained with four different digital systems, and the software modality did not influence the mutual rank of the four systems relating to their diagnostic accuracy.

Thus, the purpose of this in vitro study was to compare the efficacy of different image processing algorithms of three digital x-ray software programs (Vixwin 2000, Dimaxis and Dr. Suni Plus digital x-ray) in detecting small artificial caries defects on approximal surfaces.

\section{Materials and Methods}

\section{Preparation of samples}

The present study was approved by the ethical committee of Naresuan University, Phitsanulok, Thailand (Ethic Committee approval protocol number 5301010015). Seventy five extracted human maxillary and mandibular posterior permanent teeth from the orthodontic and periodontal treatment were used. They composed of 50 premolars and 25 molars with no restoration, fracture or caries lesion and had smooth contact surface. The 0.5 millimeter diameter high speed diamond bur was used to simulate 100 small artificial caries defects randomly. The approximal surfaces were prepared by one of the authors (Prompruk E) who was well trained and advice by the specialist from department of operative dentistry in our faculty (Figure 1). The teeth then were mounted in 25 plaster blocks which contained 2 premolars and 1 molar each. A prominent part of proximal surfaces were placed at the same vertical level to simulate normal anatomical contacts.

\section{Radiographs}

A 14 millimeters thick pink wax was placed between the tooth block and $\mathrm{x}$-ray tube to mimic the tooth-soft tissue relationship. Bitewing radiographs were taken using 3 CCD digital image systems $\left(G e n d e{ }^{\mathbb{R}}\right.$ Visualix ${ }^{\circledR}$ eHD; Gendex Dental Systems, Des Plaines, IL, USA; Planmeca Dixi 3, Planmeca Oy, Helsinki, Finland; Dr. Suni Plus, Suni Medical Imaging Inc. , California, USA) in combination with conventional intraoral x-ray machine (Gendex GX 1000 Gendex Corporation, Des Plaines, IL, USA) at $70 \mathrm{kVp}, 15$ milli-amperage for 0.08 seconds. A 12inch source-to-object distance was used with the angle between $\mathrm{x}$-ray beam and teeth long axis of 8 degrees to compensate for the slight bend of the tilt of the maxillary teeth in clinical situation (Figure 2).

\section{Dental image evaluations}

All dental images were viewed on a 19-inch monitor screen (Philips 191E1, Monitors \& Displays Taiwan Ltd., Taoyuan County, Taiwan) with help of Vixwin 2000, Dimaxis and Dr. Suni plus, which are the image enhancement software programs of the 3 digital image systems mentioned above respectively. The radiograph was enhanced with emboss, gray-scale reversed and contrast-brightness tools for

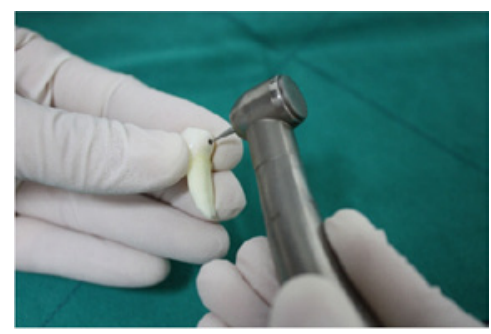

Figure 1: Artificial caries defect was prepared on the contact area or middle third of the approximal surface.

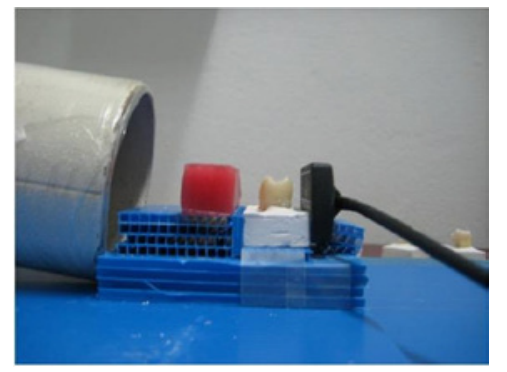

Figure 2: The relationships between tooth block, CCD, pink wax, and x-ray tube for taking bitewing radiograph. A 14 millimeters thick pink wax, cover the whole detector surface, was placed between the tooth block and $x$-ray tube to mimic the tooth-soft tissue relationship. A 12-inch source-to-object distance was used with the angle between $\mathrm{x}$-ray beam and teeth long axis of 8 degrees.

each system. Three independent observers (one oral radiologist and two sixth grade dental students) who were familiar with the digital radiography systems assessed the radiographs from each modality for presence or absence of small carious lesion on approximal surfaces. All observers successfully completed a test session before the observation analyses to ensure reader training. In clinical situation, only one observer was most familiar with embossed and grey-scale reversed digital radiograph compared with the two dental students. Thus, to avoid bias among the observers, they were prohibited to adjust the embossed and gray-scale reversed digital radiographs but they were allowed to adjust the contrast-brightness digital radiographs. Viewing sessions were performed twice for each observer, with at least a 2 weekinterval.

\section{Data analysis}

For each observer and each radiographic modality, the sensitivity, specificity, and overall accuracy were computed. The sensitivity and specificity of 3 digital $x$-ray software programs were then compared by $\mathrm{McNemar}$ test $(\mathrm{P}<0.05)$. The intraobserver and interobserver agreement were analyzed by means of Kappa statistics, interpreted according to the strength-of-agreement classification of Landis and Koch [17] : >0.81 (very good), 0.61-0.80 (good), 0.41-0.60 (moderate), $0.21-0.40$ (fair) and $<0.20$ (poor) agreements, respectively.

\section{Results}

The kappa values were $0.67-1.00$ and $0.60-0.90$ for intraobserver and interobserver agreement, respectively. All of the three observers had very good agreement with their own scorings (Table 1). Regarding emboss and contrast-brightness enhancement, the strength of agreement between the three observers were moderate good. Kappa value of the gray-scale reversed enhancement was good to very good, especially between observer 1 and observer 2 , their agreement was very good for all 3 digital systems (Table 2).

The status of the 150 proximal surfaces in 75 posterior teeth with 100 artificial carious defects was assessed. Table 3 shows the sensitivities, specificities and accuracies of 3 digital $x$-ray software programs. For the emboss enhancement, Dimaxis showed significantly higher sensitivity and accuracy than Vixwin 2000 and Dr. Suni plus $(\mathrm{P}<0.05)$. Vixwin 2000 displayed highest sensitivity of contrast-brightness enhancement for detecting caries followed by Dr. Suni plus and Dimaxis, respectively $(\mathrm{P}<0.05)$. The specificities were generally higher for all software programs. There were no significant differences between the 3 digital $\mathrm{x}$-ray software programs for gray-scale reversed enhancement $(\mathrm{P}>0.05)$. Figures 3 showed the examples of digital radiographs enhanced with 


\begin{tabular}{|l|c|c|c|}
\hline & \multicolumn{3}{|c|}{ Kappa coefficients } \\
\cline { 2 - 4 } & Observer 1 & Observer 2 & Observer 3 \\
\hline Emboss enhancement & & & \\
\hline Vixwin 2000 & 0.86 & 0.86 & 0.91 \\
\hline Dimaxis & 0.81 & 0.81 & 0.67 \\
\hline Dr.Suni plus & 0.70 & 0.70 & 0.89 \\
\hline Gray-scale reversed enhancement & & & \\
\hline Vixwin 2000 & 1.00 & 0.81 & 0.87 \\
\hline Dimaxis & 0.96 & 0.86 & 0.75 \\
\hline Dr.Suni plus & 0.99 & 0.69 & 0.95 \\
\hline Contrast-brightness enhancement & & & \\
\hline Vixwin 2000 & 0.96 & 0.85 & 0.89 \\
\hline Dimaxis & 0.96 & 0.82 & 0.81 \\
\hline Dr.Suni plus & 0.97 & 0.75 & 0.79 \\
\hline
\end{tabular}

Table 1: Intraobserver kappa coefficients by enhanced digital radiograph of the 3 digital radiographic systems.

\begin{tabular}{|l|c|c|c|}
\hline & \multicolumn{3}{|c|}{ Kappa coefficients } \\
\cline { 2 - 4 } & $\begin{array}{l}\text { Observer 1 } \\
\text { and 2 }\end{array}$ & $\begin{array}{c}\text { Observer 1 } \\
\text { and 3 }\end{array}$ & $\begin{array}{c}\text { Observer 2 } \\
\text { and 3 }\end{array}$ \\
\hline Emboss enhancement & 0.69 & 0.74 & 0.68 \\
\hline Vixwin 2000 & 0.63 & 0.60 & 0.69 \\
\hline Dimaxis & 0.61 & 0.60 & 0.71 \\
\hline Dr.Suni plus & & & \\
\hline Gray-scale reversed enhancement & 0.90 & 0.69 & 0.70 \\
\hline Vixwin 2000 & 0.84 & 0.86 & 0.82 \\
\hline Dimaxis & 0.87 & 0.76 & 0.77 \\
\hline Dr.Suni plus & & & \\
\hline Contrast-brightness enhancement & 0.79 & 0.66 & 0.66 \\
\hline Vixwin 2000 & 0.72 & 0.75 & 0.71 \\
\hline Dimaxis & 0.66 & 0.65 & 0.61 \\
\hline Dr.Suni plus & & & \\
\hline
\end{tabular}

Table 2: Interobserver kappa coefficients by enhanced digital radiograph of the 3 digital radiographic systems.

the 3 digital x-ray software programs.

\section{Discussion}

The digital radiographs in the present study were evaluated by 3 observers which composed of 1 oral radiologist and 2 dental students. Table 2 showed slightly moderate kappa values between observers for emboss and contrast-brightness enhancement, even though a test session before the observation assessments to ensure reader training was performed and the test result were excellent. Table 2 showed slightly moderate kappa values between observers for emboss and contrastbrightness enhancement that were in accordance with Langlais et al. [18] who found that a high level of observer agreement was difficult to obtain in the case of caries limited to the enamel, although they studied in natural carious lesion. Kang et al. [19] carried out a phantom study and reported that the interexaminer reproducibility (kappa value) was 0.43 for detecting the proximal defect and caries. Factors that may influence the interobserver agreements include scale contrast, time, duration for image evaluation, experience and familiarity of the observers with the digital radiographic systems.

Many authors have investigated diagnostic accuracy of digital radiography in detecting dental caries. However, they always compare between conventional digital radiograph and film-based radiographs, or among various enhanced radiographs within the same digital program $[3,4,20,21]$. The present study compared the efficiency of various image processing programs for the detection of small proximal carious lesion between 3 digital radiographic system, namely, Vixwin
2000, Dimaxis, and Dr. Suni plus. According to table 3, the sensitivities in detecting small dental caries on proximal surfaces by using emboss enhancement of the 3 programs are lower than those of gray-scale reversed and contrast-brightness enhancement as a result of the high false negative values. Owing to the large size of proximal surfaces of posterior teeth and the subtle mineral loss initially presented by lesions on these surfaces, small proximal caries on posterior teeth are usually very difficult to visualize on radiographs, especially for the one who is not familiar with digital radiographic software programs or insufficient experience. Regarding the use of digital radiographic programs in our dental school, emboss enhancement program is less used and less popular compare with the other two programs (gray-scale reversed and contrast-brightness enhancement). This may be one of the reasons of its low sensitivity. Pontual et al. [14] reported that natural enamel lesions have low contrast and are not sharply defined that lead to difficulty in detection on conventional radiographs. Although it was not used natural enamel carious lesions in the present investigation, their results are consistent with ours. It seems that high contrast is a prerequisite for interpretation of radiographic proximal caries. Radiographs need to be quite dark with good contrast to provide an optimal basis for caries detection.

Diagnostic accuracy of emboss-enhanced images in Dimaxis was significantly higher than Vixwin 2000 and Dr. Suni plus $(\mathrm{P}<0.05 ; 74 \%$, $64 \%$, and $60 \%$, respectively), which were not found in that of grayscale reversed and contrast-brightness-enhanced images. However, diagnostic accuracies in detecting small proximal caries using grayscale reversed and contrast-brightness-enhancement were higher than that of emboss enhancement $(89.33 \%-90 \%$, and $84.67 \%-89.33 \%$, respectively) (Table 3). Specificities were generally higher for all software programs (ranging from $86 \%$ to $96 \%$ ). A caries diagnostic method which emphasizes specificity at the expense of some loss of sensitivity seems preferable, as the consequences of false-positive results in clinical terms may be the unnecessary filling of sound teeth and the initiation of a continuous and increased repair cycle. The clinical decision whether to restore caries should be made based on cavitation rather than histological lesion depth [22]. The first level of the decision process is to determine the state of the tooth surface, whether sound, having a lesion or being filled. If there is a lesion, one may assess the activity state of the lesion. The last step is to generate a proper treatment decision. Active lesions demand professional

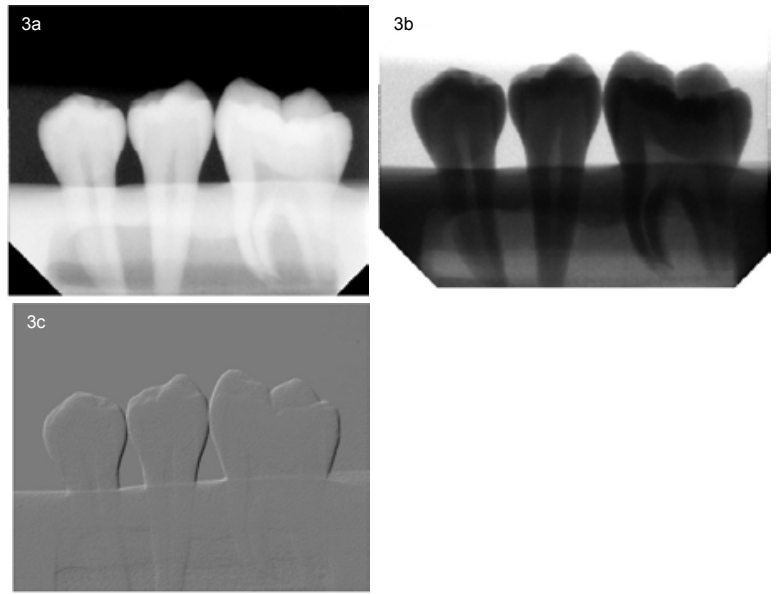

Figure 3: Examples of digital radiographs enhanced with the 3 digital $x$-ray software programs; a: contrast-brightness-enhanced image of Vixwin 2000, b: gray-scale reversed enhanced image of Dimaxis, c: emboss enhanced image of Dr.Suni plus. 


\begin{tabular}{|c|c|c|c|c|c|c|c|}
\hline & \multicolumn{4}{|c|}{ No. of case } & \multirow{2}{*}{$\begin{array}{c}\text { Sensitivity } \\
\%\end{array}$} & \multirow{2}{*}{$\begin{array}{c}\text { Specificity } \\
\%\end{array}$} & \multirow{2}{*}{$\begin{array}{c}\text { Accuracy } \\
\%\end{array}$} \\
\hline & TP & $\mathrm{TN}$ & $\mathrm{FP}$ & $\mathrm{FN}$ & & & \\
\hline \multicolumn{8}{|c|}{ Emboss enhancement } \\
\hline Vixwin 2000 & 50 & 46 & 4 & 50 & $50.00^{*}$ & 92.00 & $64.00^{*}$ \\
\hline Dimaxis & 68 & 43 & 7 & 32 & $68.00^{*},+$ & 86.00 & $74.00^{*},+$ \\
\hline Dr.Suni plus & 42 & 48 & 2 & 58 & $42.00^{\dagger}$ & 96.00 & $60.00^{\dagger}$ \\
\hline \multicolumn{8}{|c|}{ Gray-scale reversed enhancement } \\
\hline Vixwin 2000 & 89 & 46 & 4 & 11 & 89.00 & 92.00 & 90.00 \\
\hline Dimaxis & 89 & 46 & 4 & 11 & 89.00 & 92.00 & 90.00 \\
\hline Dr.Suni plus & 89 & 45 & 5 & 11 & 89.00 & 90.00 & 89.33 \\
\hline \multicolumn{8}{|c|}{ Contrast-brightness enhancement } \\
\hline Vixwin 2000 & 91 & 43 & 7 & 9 & $91.00^{*}$ & 86.00 & 89.33 \\
\hline Dimaxis & 81 & 46 & 4 & 19 & $81.00^{*}+\dagger$ & 92.00 & 84.67 \\
\hline Dr.Suni plus & 84 & 46 & 4 & 16 & $84.00^{\dagger}$ & 92.00 & 86.67 \\
\hline
\end{tabular}

${ }^{*}+\mathrm{P}<0.05$ TP: true positive, TN: true negative, FP: false positive, FN: false negative

Table 3: Sensitivity, specificity, and diagnostic accuracy of enhanced digital radiographic images.

treatment [23]. For example, in case of a deep dentin carious lesion seen on radiograph, but it is not clinically cavitated, the lesion need for restoration.

Although the results of previous $[3,11,12,14,24]$ indicated that diagnostic accuracy of intraoral digital radiograph is comparable to film-based radiograph, these studies were performed in vitro. The results of the present study was also indicated same as the formers. However, we used artificial caries defects created by a bur, not natural caries lesion. Thus these studies results are still questionable that they can be used as represent data of clinical situation or not. The further clinical study is need for more reliability and reproducibility. There were some limitations of the present study, such as using artificial instead natural carious lesions. With digital radiography, natural carious lesion showed as a gradual transition between the lesion core and the surrounding sound enamel, whereas artificial lesions created with a bur showed a well-defined border. Although not reproducing the histology condition of dental caries, mechanically created defects or "artificial dental caries" have been used in a number of recent studies [19,25].

The movement from analog films towards digital imaging systems is based on a number of advantages of digital imaging systems including the development of the sensors. These technologies have undergone considerable improvement and many other systems have emerged that included CCD (charge-coupled device), PSP (photostimulable phosphor), and CMOS (complementary metal oxide semiconductor) designs. Sensors with dimensions suitable for use in the mouth have been developed due to progress in minimization of electronic circuitry [26]. The various digital systems display the images in the systems' own software, which often differ significantly in relation to design, userfriendliness, number and quality of facilities for image enhancement [16]. In dental schools or hospitals, where several digital radiography systems are in use for dental examinations, it is widespread for all images, irrespective of origin, to be assessed using general software offering the most prevalent facilities for image enhancement [27]. Such a procedure is used because it is too inconvenient for the observer to check which radiographic systems have been used for different images included in an examination and thereafter assess the various images in their respective software modalities. The use of universal software for displaying images from all systems in use in larger departments is, of course, performed on the assumption that the image quality and diagnostic accuracy are not harmed [16].

In conclusion, the efficiency of Dimaxis's emboss enhancement digital radiograph was higher than Vixwin 2000 and Dr. Suni plus significantly. There was no significant difference of the gray-scale reverse and contrast-brightness's enhancement efficiency. The artificial small proximal caries made in the present study may be considered as the clinical situation because of the low detecting threshold of human eyes for small artificial or natural carious lesions. Sometimes very small (natural or artificial) or early carious lesions are invisible to the naked eyes. This study results may be useful for dentist in considering the use of various software programs for detecting small carious lesion on proximal tooth surface in addition to increase their experience and familiarity.

\section{References}

1. Hintze H, Wenzel A, Jones C (1994) In vitro comparison of D- and E-speed film radiography, $\mathrm{RVG}$, and visualix digital radiography for the detection of enamel approximal and dentinal occlusal caries lesions. Caries Res 28: 363-367.

2. Hintze H, Wenzel A, Frydenberg M (2002) Accuracy of caries detection with four storage phosphor systems and E-speed radiographs. Dentomaxillofac Radiol 31: 170-175.

3. Castro VM, Katz JO, Hardman PK, Glaros AG, Spencer P (2007) In vitro comparison of conventional film and direct digital imaging in the detection of approximal caries. Dentomaxillofac Radiol 36: 138-142.

4. Shi XQ, Li G (2009) Detection accuracy of approximal caries by black-andwhite and color-coded digital radiographs. Oral Surg Oral Med Oral Pathol Oral Radiol Endod 107: 433-436.

5. Haiter-Neto F, dos Anjos Pontual A, Frydenberg M, Wenzel A (2008) Detection of non-cavitated approximal caries lesions in digital images from seven solidstate receptors with particular focus on task-specific enhancement filters. An ex vivo study in human teeth. Clin Oral Investig 12: 217-223.

6. Wenzel A (1995) Current trends in radiographic caries imaging. Oral Surg Ora Med Oral Pathol Oral Radiol Endod 80: 527-539.

7. Haak R, Wicht MJ, Noack MJ (2001) Conventional, digital and contrastenhanced bitewing radiographs in the decision to restore approximal carious lesions. Caries Res 35: 193-199.

8. Tyndall DA, Ludlow JB, Platin E, Nair M (1998) A comparison of Kodak Ektaspeed Plus film and the Siemens Sidexis digital imaging system for caries detection using receiver operating characteristic analysis. Oral Surg Oral Med Oral Pathol Oral Radiol Endod 85: 113-118.

9. Senel B, Kamburoglu K, Uçok O, Yüksel SP, Ozen T, et al. (2010) Diagnostic accuracy of different imaging modalities in detection of proximal caries. Dentomaxillofac Radiol 39: 501-511.

10. Lehmann TM, Troeltsch E, Spitzer K (2002) Image processing and enhancement provided by commercial dental software programs. Dentomaxillofac Radiol 31 264-272.

11. Syriopoulos K, Sanderink GC, Velders XL, van der Stelt PF (2000) Radiographic detection of approximal caries: a comparison of dental films and digital imaging systems. Dentomaxillofac Radiol 29: 312-318. 
Citation: Tantanapornkul W, Mongkolrop P, Manoping P, Hannanta-anant A, Prompruk E (2012) Efficacy of Different Image Processing Programs in Detecting Small Artificial Caries Defects on Approximal Surfaces. Dentistry 2:129. doi:10.4172/2161-1122.1000129

Page 5 of 5

12. Hintze H, Wenzel A (2002) Influence of the validation method on diagnostic accuracy for caries. A comparison of six digital and two conventional radiographic systems. Dentomaxillofac Radiol 31: 44-49.

13. Haiter-Neto F, dos Anjos Pontual A, Frydenberg M, Wenzel A (2007) A comparison of older and newer versions of intraoral digital radiography systems: diagnosing noncavitated proximal carious lesions. J Am Dent Assoc 138: $1353-1359$

14. Pontual AA, de Melo DP, de Almeida SM, Bóscolo FN, Haiter Neto F (2010) Comparison of digital systems and conventional dental film for the detection of approximal enamel caries. Dentomaxillofac Radiol 39: 431-436.

15. Gotfredsen E, Wenzel A, Gröndahl HG (1996) Observers' use of image enhancement in assessing caries in radiographs taken by four intra-oral digital systems. Dentomaxillofac Radiol 25: 34-38.

16. Hintze $H$ (2006) Diagnostic accuracy of two software modalities for detection of caries lesions in digital radiographs from four dental systems. Dentomaxillofac Radiol 35: 78-82

17. Landis JR, Koch GG (1977) The measurement of observer agreement for categorical data. Biometrics 33: 159-174.

18. Langlais RP, Skoczylas LJ, Prihoda TJ, Langland OE, Schiff T (1987) Interpretation of bitewing radiographs: application of the kappa statistic to determine rater agreements. Oral Surg Oral Med Oral Pathol 64: 751-756.

19. Kang BC, Farman AG, Scarfe WC, Goldsmith LJ (1996) Observer differentiation of proximal enamel mechanical defects versus natural proximal dental caries with computed dental radiography. Oral Surg Oral Med Oral Pathol Oral Radiol Endod 82: 459-465.
20. Møystad A, Svanaes DB, van der Stelt PF, Gröndahl HG, Wenzel A, et al (2003) Comparison of standard and task-specific enhancement of Digora storage phosphor images for approximal caries diagnosis. Dentomaxillofac Radiol 32: 390-396.

21. Haak R, Wicht MJ (2005) Grey-scale reversed radiographic display in the detection of approximal caries. J Dent 33: 65-71.

22. Young DA, Featherstone JD (2005) Digital imaging fiber-optic trans-illumination, F-speed radiographic film and depth of approximal lesions. J Am Dent Assoc 136: $1682-1687$

23. Nyvad B (2004) Diagnosis versus detection of caries. Caries Res 38: 192-198.

24. Peker I, Toraman Alkurt M, Altunkaynak B (2007) Film tomography compared with film and digital bitewing radiography for proximal caries detection. Dentomaxillofac Radiol 36: 495-499.

25. Kang BC, Goldsmith LJ, Farman AG (1998) Observer differentiation of mechanical defects versus natural dental caries cavitations on monitordisplayed images with imaging plate readout. Oral Surg Oral Med Oral Pathol Oral Radiol Endod 86: 595-600.

26. Wakoh M, Kuroyanagi K (2001) Digital imaging modalities for dental practice Bull Tokyo Dent Coll 42: 1-14.

27. Gotfredsen E, Wenzel A (2003) Integration of multiple direct digital imaging sources in a picture archiving and communication system (PACS). Dentomaxillofac Radiol 32: 337-342. 